\title{
Aging and Memory Effects in Superparamagnets and Superspin Glasses
}

\author{
M. Sasaki, P. E. Jönsson, and H. Takayama \\ Institute for Solid State Physics, University of Tokyo, \\ 5-1-5 Kashiwa-no-ha, Kashiwa, Chiba 277-8581, Japan \\ H. Mamiya \\ National Institute for Materials Science, Sengen 1-2-1, Tsukuba, Ibaraki 305-0047, Japan
}

(Dated: October 3, 2018)

\begin{abstract}
Many dense magnetic nanoparticle systems exhibit slow dynamics which is qualitatively indistinguishable from that observed in atomic spin glasses and its origin is attributed to dipole interactions among particle moments (or superspins). However, even in dilute nanoparticle systems where the dipole interactions are vanishingly small, slow dynamics is observed and is attributed solely to a broad distribution of relaxation times which in turn comes from that of the anisotropy energy barriers. To clarify characteristic differences between the two types of slow dynamics, we study a simple model of a non-interacting nanoparticle system (a superparamagnet) analytically as well as ferritin (a superparamagnet) and a dense Fe-N nanoparticle system (a superspin glass) experimentally. It is found that superparamagnets in fact show aging (a waiting time dependence) of the thermoremanent-magnetization as well as various memory effects. We also find some dynamical phenomena peculiar only to superspin glasses such as the flatness of the field-cooled magnetization below the critical temperature and memory effects in the zero-field-cooled magnetization. These dynamical phenomena are qualitatively reproduced by the random energy model, and are well interpreted by the so-called droplet theory in the field of the spin-glass study.
\end{abstract}

\section{INTRODUCTION}

One of the most attractive topics in the field of condensed matter physics is slow dynamics such as nonexponential relaxation, aging (a waiting time dependence of observables),$\frac{1,2}{, 2}$ and memory effects. These phenomena are observed in various systems like polymers high- $T_{\mathrm{c}}$ super-conductors,$\frac{5}{\underline{5}}$ granular materials $\frac{6}{\underline{6}}$ and spin glasses. Especially, in the field of spin glasses, slow dynamics has been studied widely both experimentally 2.7 .8 .9 and theoretically $10,11,12,13,14,15$ to examine the validity of novel concepts of spin glasses such as a hierarchical organization of states 16.17 and temperature chaos $\underline{18.19 .20}$ These extensive studies have revealed various interesting behavior in dynamics like coexistence of memory and rejuvenation ${ }^{7.8 .9}$ Such findings have stimulated many researchers to study slow dynamics in various systems like geometrically frustrated magnets $\stackrel{21.22}{\longleftarrow}$ transition-metal

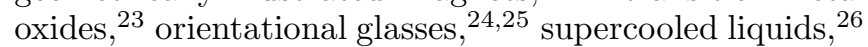
and dense magnetic nanoparticle systems ${ }^{27,28,29,30,31.32}$ by using experimental protocols developed in the study of spin glasses. Magnetic nanoparticle systems, which we study in this paper, are of current interest because of their significance for technological applications as well as for their fundamental magnetic properties ${ }^{33}$

In magnetic nanoparticle systems, there are two possible origins of slow dynamics. The first one is a broad distribution of relaxation times originating solely from that of the anisotropy energy barriers of each nanoparticle moment. This is the only source of slow dynamics for sparse (weakly interacting) magnetic nanoparticle systems, in which the nanoparticles are fixed in space. We hereafter call magnetic moments of each nanoparticle superspins, and such weakly interacting magnetic nanopar- ticle systems superparamagnets. However, for dense magnetic nanoparticle systems, there is a second possible origin of slow dynamics, namely, cooperative spin glass dynamics due to frustration caused by strong dipolar interactions among the particles and randomness in the particle positions and anisotropy axes orientations $\underline{34}$ In fact, evidences for a spin glass transition such as the critical divergence of the nonlinear susceptibility have been found in dense magnetic nanoparticle systems ${ }^{35.36 .37} \mathrm{We}$ hereafter call such dense magnetic nanoparticle systems, which exhibit spin glass behavior, superspin glasses.

Now the point is that magnetic nanoparticle systems involve two possible mechanisms for slow dynamics, and which of the two is relevant depends essentially on the concentration of nanoparticles. Then, in order to understand appropriately slow dynamics in magnetic nanoparticle systems, it is desirable to clarify which observed phenomena are simply due to slow dynamics caused by a broad distribution of relaxation times, and which ones are brought by cooperative dynamics peculiar to superspin glasses. For this purpose, we first study a simple model of non-interacting magnetic nanoparticle systems (superparamagnets) analytically. As a consequence, we find that even superparamagnets exhibit aging of the thermoremanent-magnetization and various memory effects. In particular, we show that the curious memory effects recently reported by Sun et al., 38 which were claimed to give evidences of the existence of a superspin glass phase, can be understood simply as superparamagnetic relaxation (see also Refs. 394041 .)

We also perform experiments on a ferritin (a superparamagnet ${ }^{42.43}$ ) and a dense $\mathrm{Fe}-\mathrm{N}$ nanoparticle systems (a superspin glass 28.36 .44 .45 ). The results of ferritin are qualitatively similar to those of our simple model 
of superparamagnets. The comparison of the phenomena observed in the superparamagnet and the superspin glass reveals some properties peculiar only to superspin glasses, e.g., the flatness of the field-cooled magnetization below the critical temperature and memory effects in the zero-field-cooled magnetization. Particularly, the former phenomenon reminds us of Parisi's equilibrium susceptibility in the spin-glass mean-field theory ${ }^{46}$ However, we propose an interpretation based on the spinglass droplet theory ${ }^{20.47}$ which predicts the instability of the spin-glass phase under a static magnetic field of any strengths and so claims the observed field-cooled magnetization to be a property far from equilibrium 48 We also show that these experimental results peculiar to superspin glasses are qualitatively reproduced by the random energy model $\stackrel{49,50,51}{ }$

The outline of the present manuscript is as follows. In section [I] we introduce a model of superparamagnets and report aging and memory effects observed in this model. The results of experiments on ferritin are also shown in this section. In section 11 we show experimental results on a dense $\mathrm{Fe}-\mathrm{N}$ nanoparticle system. Some properties found only in the superspin glass are interpreted by the random energy model and the droplet theory. Section IV is devoted for summary.

\section{SLOW DYNAMICS IN SUPERPARAMAGNETS}

\section{A. Model and master equation approach}

Here we adopt a simple model which is considered to describe the essential slow dynamics in non-interacting magnetic nanoparticle systems (superparamagnets). The magnetic moment (superspin) of one nanoparticle, which does not interact with any other superspins, is supposed to occupy one of two states with energies $-K V \pm h M_{\mathrm{s}} V$, where $K$ is the bulk anisotropy constant, $V$ the volume of the nanoparticle, $h$ an applied field in linear response regime, and $M_{\mathrm{s}}$ the saturation magnetization. Here we supposed that the direction of the field is parallel to the anisotropy axes for simplicity. The superparamagnetic relaxation time in zero field for the thermal activation over the energy barrier $K V$ is given by $\tau=\tau_{0} \exp (K V / T)$, where $\tau_{0}$ is a microscopic time.

The occupation probability of one of the two states, in which the superspin is in parallel (antiparallel) to the field direction, is denoted by $p_{1}(t)\left(1-p_{1}(t)\right)$, and is solved by the following master equation ${ }^{52}$

$$
\frac{d}{d t} p_{1}(t)=-W_{1 \rightarrow 2}(t) p_{1}(t)+W_{2 \rightarrow 1}(t)\left\{1-p_{1}(t)\right\},
$$

where $W_{1 \rightarrow 2}(t)\left(W_{2 \rightarrow 1}(t)\right)$ is the transition rate from the state 1 to 2 (2 to 1 ) at time $t$. To the leading order in $h(t)$ they are written as

$$
W_{1 \rightarrow 2}(t)=\frac{1}{2} \tau_{0}^{-1} \exp [-K V / T(t)]\left\{1-M_{\mathrm{s}} V h(t) / T(t)\right\},(2)
$$

$$
W_{2 \rightarrow 1}(t)=\frac{1}{2} \tau_{0}^{-1} \exp [-K V / T(t)]\left\{1+M_{\mathrm{s}} V h(t) / T(t)\right\} .(3)
$$

The above master equation can be solved analytically for any temperatures and field protocols represented by $T(t)$ and $h(t)$ from a given initial condition, and the magnetization of the particle with volume $V$ is given by

$$
M(t ; V)=\left[2 p_{1}(t ; V)-1\right] M_{\mathrm{s}} V .
$$

For example, in the case that $h(t)=h$ and $T(t)=T$, we obtain

$$
\begin{aligned}
M(t ; V)= & M(t=0 ; V) \exp (-t / \tau) \\
& +\frac{\left(M_{\mathrm{s}} V\right)^{2} h}{T}\{1-\exp (-t / \tau)\},
\end{aligned}
$$

where $\tau \equiv \tau_{0} \exp (K V / T)$. Note that the additional condition $h=0$ leads us to the familiar formulation for the decay of the thermoremanent-magnetization.

From Eqs.(1-4), we notice that $p_{1}(t)=1 / 2(M(t)=0)$ at any $t$ if $p_{1}(0)=1 / 2$ and $h(t)=0$. This means that in any genuine zero-field-cooled (ZFC) processes starting from $M=0, p_{1}(t)$ is independent of the schedule of temperature change $T(t)$, i.e., no memory is imprinted in the process. Experimentally, a demagnetized initial state is obtained by choosing the starting temperature sufficiently high.

The total magnetization of the nanoparticle system is evaluated by averaging over the volume distribution,

$$
\bar{M}(t)=\int d V P(V) M(t ; V) \equiv \int d V M_{\mathrm{spec}}(t ; V) .
$$

Here, the integrand (the $M$-spectrum) denoted as $M_{\text {spec }}(t ; V)$ plays an important role in the arguments below. For the explicit evaluation of $\bar{M}(t)$, we use a lognormal distribution

$$
P(V)=\exp \left[-\ln (V)^{2} /\left(2 \gamma^{2}\right)\right] /(\gamma V \sqrt{2 \pi}),
$$

with $\gamma=0.6$. Although quantitative and some minute qualitative results may depend on the value of $\gamma$, the functional form of $P(V)$, and even our basic assumption of the two-states representation, we do not go into such details here, expecting that our simplest model catches up the essence of slow dynamics of superparamagnets.

In the present work the average anisotropic energy $K \bar{V}$ is chosen as the unit of energy as well as that of temperature by setting $k_{\mathrm{B}}=1 . V$ is measured in unit of the average volume $\bar{V}$, which for the log-normal distribution [Eq. (7)] is given by $\exp \left(\gamma^{2} / 2\right)$. As for the time-scale, we suppose that the microscopic time $\tau_{0}$ for superspins of realistic nanoparticles is around $10^{-9} \mathrm{~s}$, and that a typical experimental time window is around $10^{2} \mathrm{~s}$. We therefore investigate our model in the time window around $10^{11} \tau_{0}$ expecting that it corresponds to typical experimental time scales. 


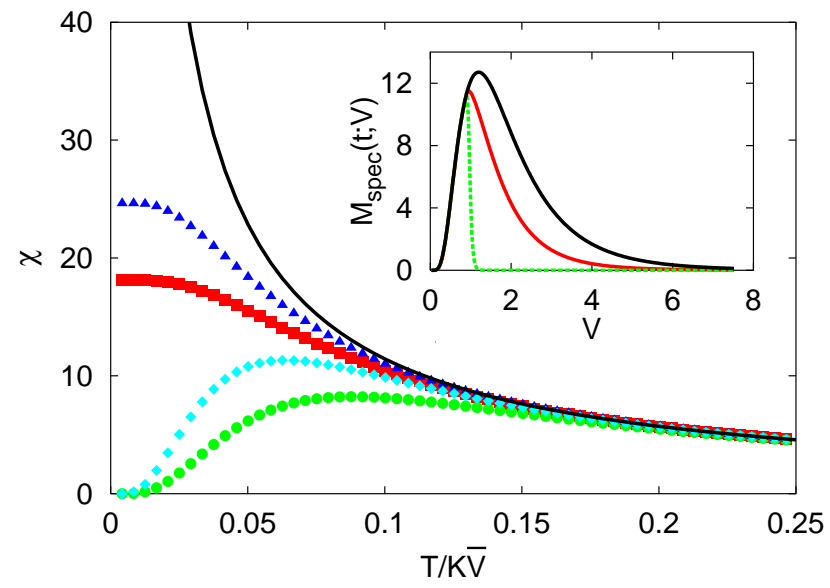

FIG. 1: (Color online) ZFCM/FCM with the cooling rate $r=$ $2.4 \cdot 10^{12} \tau_{0}$ per temperature unit (circles/squares) and those with slower cooling rate $r=2.4 \cdot 10^{16} \tau_{0}$ (diamonds/triangles). The line is the susceptibility in equilibrium (the Curie law). The inset shows the $M$-spectra of the ZFCM, FCM and the magnetization in equilibrium at $T=0.042$ (from left to right). The cooling rate for $\mathrm{ZFCM} / \mathrm{FCM}$ is $2.4 \cdot 10^{12} \tau_{0}$.

\section{B. ZFC and FC magnetizations}

Let us begin our arguments from the most fundamental and well-known protocols, i.e., the measuring processes of the zero-field-cooled magnetization (ZFCM) and the field-cooled magnetization (FCM). In the ZFC process, the system is rapidly cooled to a low temperature in zero field, and then the induced magnetization by an applied field $h$ is measured as the temperature is gradually increased. In the FC process, on the other hand, the system is gradually cooled under $h$ from a sufficiently high temperature so that the system is in equilibrium at the highest temperature. The circles/squares in Fig. 11 represent the ZFCM/FCM observed with heating/cooling rate, $r$, of $2.4 \times 10^{12} \tau_{0}$ per temperature unit ${ }^{59}$ As usually adopted, the peak position of the ZFCM is regarded as the blocking temperature, $T_{\mathrm{B}}$, which is $\simeq 0.088$ for the present process. If the rate $r$ is $10^{4}$ times slower, we obtain $T_{\mathrm{B}} \simeq 0.063$ (diamonds for the ZFCM and triangles for the FCM). If we make $r$ infinitely slow, both the ZFCM and the FCM curves coincide with the one given by the Curie law.

In the inset of Fig. 1 we show the $M$-spectra (the integrand of Eq.(6)) of the ZFCM, FCM and the magnetization in equilibrium at $T=0.042$ (from left to right). One can clearly see that the parts of the three $M$-spectra for $V$ smaller than a certain value, which we denote as $V_{\mathrm{B}}$, lie on top of each other. This means that superspins of these small nanoparticles are equilibrated within the characteristic time-scale of the cooling/heating process. On the other hand, the $M$-spectrum of the ZFCM at $V \gtrsim V_{\mathrm{B}}$ is zero, indicating that superspins of these larger nanoparticles are still blocked to their initial values. We call $V_{\mathrm{B}}$ the blocking volume which depends strongly (lin- early) on $T$ and weakly (logarithmically) on the observation time-scale. Also we call superspins of nanoparticles with $V \lesssim V_{\mathrm{B}}, V \simeq V_{\mathrm{B}}$, and $V \gtrsim V_{\mathrm{B}}$ superparamagnetic, dynamically active, and blocked or frozen, respectively.

By passing we emphasize another characteristic feature of the FCM in superparamagnets. Namely, the FCM always increases as the temperature is decreased. This is simply because superspins are blocked (or frozen) in the direction of the field.

\section{Aging and memory effects}

Let us now consider the thermoremanentmagnetization (TRM) protocol, where we cool the system in a field $h$ at a certain rate, stop the cooling at
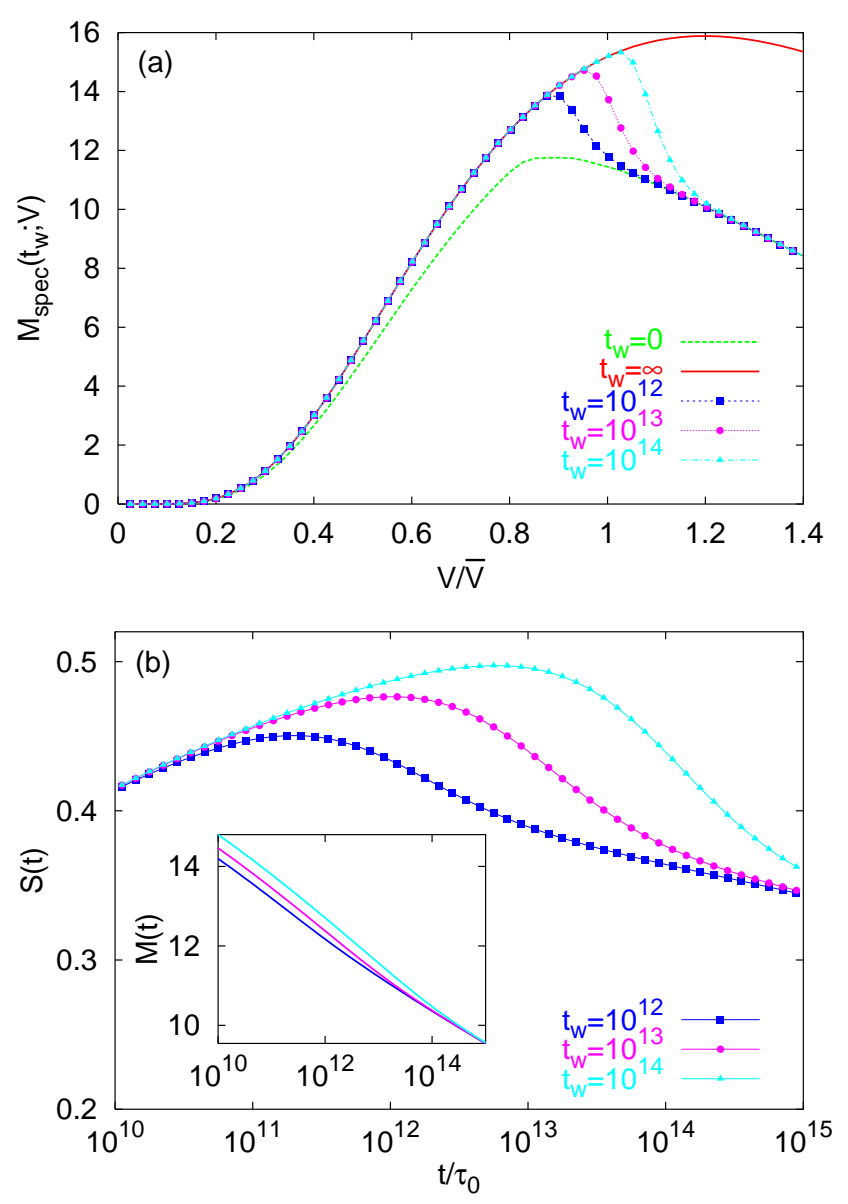

FIG. 2: (Color online) (a) $M_{\mathrm{spec}}\left(t_{\mathrm{w}} ; V\right)$ of the FC process. The system is cooled to $T_{\mathrm{m}}(=0.033)$ at the rate of $2.4 \cdot 10^{12} \tau_{0}$ per temperature unit, and is kept at $T_{\mathrm{m}}$ for $t_{\mathrm{w}}$. The field is applied in the whole process. (b) Susceptibility $\chi_{\mathrm{TRM}}\left(t, t_{\mathrm{w}}\right)$ measured in the TRM protocol (inset) and its logarithmic time derivative $S(t) \equiv-d \log \chi_{\mathrm{TRM}}\left(t, t_{\mathrm{w}}\right) / d \log t$ (main frame) vs t, where $t$ is the elapsed time after the field is cut. The cooling rate and $T_{\mathrm{m}}$ are the same as in a). In the inset, the corresponding waiting time increases from left to right. 
a measurement temperature, $T_{\mathrm{m}}$, let the system relax for a waiting time of $t_{\mathrm{w}}$, and then cut the field and observe the magnetization decay. During the FC aging before cutting the field, the parts of the $M$-spectrum for the frozen and superparamagnetic superspins, do not change significantly, while that of the dynamically active superspins does change as seen in Fig. 2(a). The peak of the $M$-spectrum shifts to larger volumes with increasing $t_{\mathrm{w}}$. The peak position appears around $V^{*}$ where the corresponding relaxation time $\tau_{0} \exp \left(K V^{*} / T_{\mathrm{m}}\right)$ is comparable with $t_{\mathrm{w}}$. This naturally means that the TRM decreases most rapidly when the time $t$ elapsed after the field is cut is nearly equal to $t_{\mathrm{w}}$. Indeed, Fig. 2(b) shows that the relaxation rate $S(t) \equiv-h^{-1} d \log M / d \log t$ in the TRM protocol has a peak around $t_{\mathrm{w}}$. Thus we conclude that aging (a $t_{\mathrm{w}}$-dependence) of the TRM does exist even in superparamagnets.

As mentioned in subsection $1 \mathrm{~A}$ however, the ZFCM curve is independent of $t_{\mathrm{w}}$. One may consider that this $t_{\mathrm{w}}$-independence of the ZFCM is a consequence of the simple two-states description of our model. Actually, by considering several competing sources of anisotropy (for instance magnetocrystalline and magnetostatic energy), we can think of a multi-states system with some energy levels different from each other. Then, the ZFCM of the model should depend weakly on $t_{\mathrm{w}}$ even if interactions among particles are absent. In fact, we will show in section III that the random energy model, which has a huge number of states whose energies are different from each other, exhibits strong aging in genuine

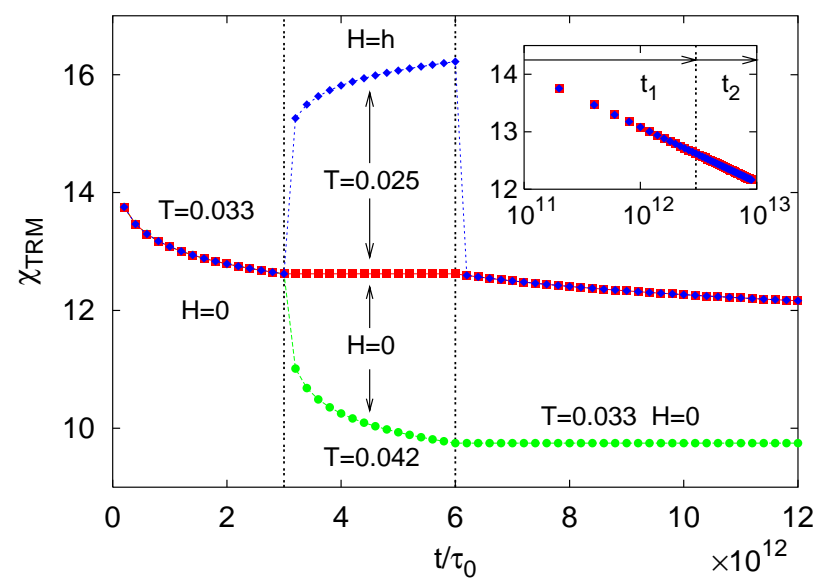

FIG. 3: (Color online) $\chi_{\mathrm{TRM}}$ vs time using the same protocols as in Figs. 3,4,5 of Sun et al ${ }^{38}$. The system is cooled to $T=0.033$ at the rate of $2.4 \cdot 10^{13} \tau_{0}$ per temperature unit in a field which is cut just before recording $\chi_{\mathrm{TRM}}$. After a time of $t_{1}=3 \times 10^{12} \tau_{0}$ the temperature is changed. The relaxation at the new temperature is recorded either in $H=0$ or $H=h$ in period of $t_{2}=3 \times 10^{12} \tau_{0}$. Then the temperature is shifted back to $T=0.033$ and the field is set to zero. In the inset, $t_{1}$ and $t_{3}$ parts of $\chi_{\mathrm{TRM}}$ with the negative temperature cycling are plotted as a function of the total time elapsed at $T=0.033$.
ZFC protocols. However, we consider that a significantly small $t_{\mathrm{w}}$-dependence of the ZFCM as compared to that of the TRM is one of the characteristic properties of superparamagnets since in ordinary spin glasses, a strong $t_{\mathrm{w}}$-dependence is observed not only in the TRM but also in the ZFCM. Therefore, indubitable experimental evidence for spin-glass dynamics in a system can only be found by investigating aging effects in the ZFCM.

From the sum rule for the ZFCM, TRM and FCM, we find

$$
M_{\mathrm{TRM}}\left(t, t_{\mathrm{w}}\right)=M_{\mathrm{FC}}\left(t+t_{\mathrm{w}}\right)-M_{\mathrm{ZFC}}(t),
$$

where we have used the fact that the ZFCM does not depend on $t_{\mathrm{w}}$ in our model. This equation tells us that the $t_{\mathrm{w}}$-dependence of the TRM in our model is merely a consequence of slow relaxation of the FCM. This is in contrast to ordinary spin glasses where the TRM and the ZFCM strongly depend on $t_{\mathrm{w}}$ even if $M_{\mathrm{FC}}\left(t^{\prime}\right)$ for $t^{\prime} \geq t_{\mathrm{w}}$ hardly relaxes. 53

Another important point is that the peak position of the $M$-spectrum in Fig. 2(a) (and the relaxation rate $S(t)$ in Fig. 2(b)) ceases to shift if $t_{\mathrm{w}} \geq \tau_{0} \exp \left(K V_{\text {peak }} / T_{\mathrm{m}}\right)$, where $V_{\text {peak }}$ is the peak position of the $M$-spectrum in equilibrium (its explicit value is around 1.2 in the present case). On the other hand, aging in spin glasses is believed to persist eternally in the thermodynamic limit since the relaxation time diverges below the critical temperature.

After the field is cut in the above-mentioned TRM protocol with $t_{\mathrm{w}}=0$, we may further introduce some cycling processes,, 30.38 as shown in Fig. 3] Now let us first consider a negative-temperature cycling in zero field. The temperature is changed as $T_{\mathrm{m}}=0.033 \rightarrow T_{2}=0.025 \rightarrow$

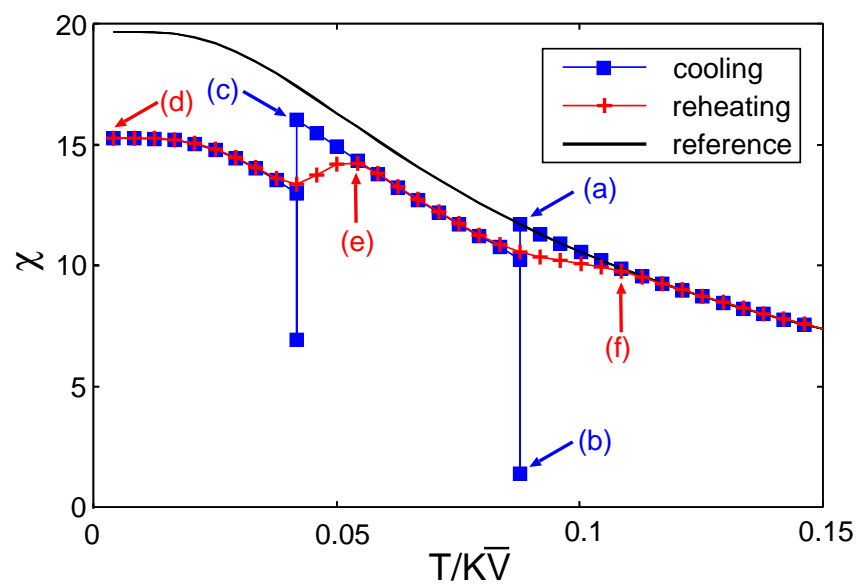

FIG. 4: (Color online) FC susceptibility vs temperature observed in the same protocol as in Fig. 2 of Sun et al ${ }^{38}$. The field is cut during the intermittent stops of the cooling at $T_{1}=0.088$ and at $T_{2}=0.042$ for a period of $10^{14} \tau_{0}$. The magnetization in zero field after the waiting time is shown although it was not shown by Sun et al. The arrows in the figure indicate at which stages during the procedure we measure and show the $M$-spectra in Fig. 5 The cooling (and reheating) rate is the same as that in Fig. 3 


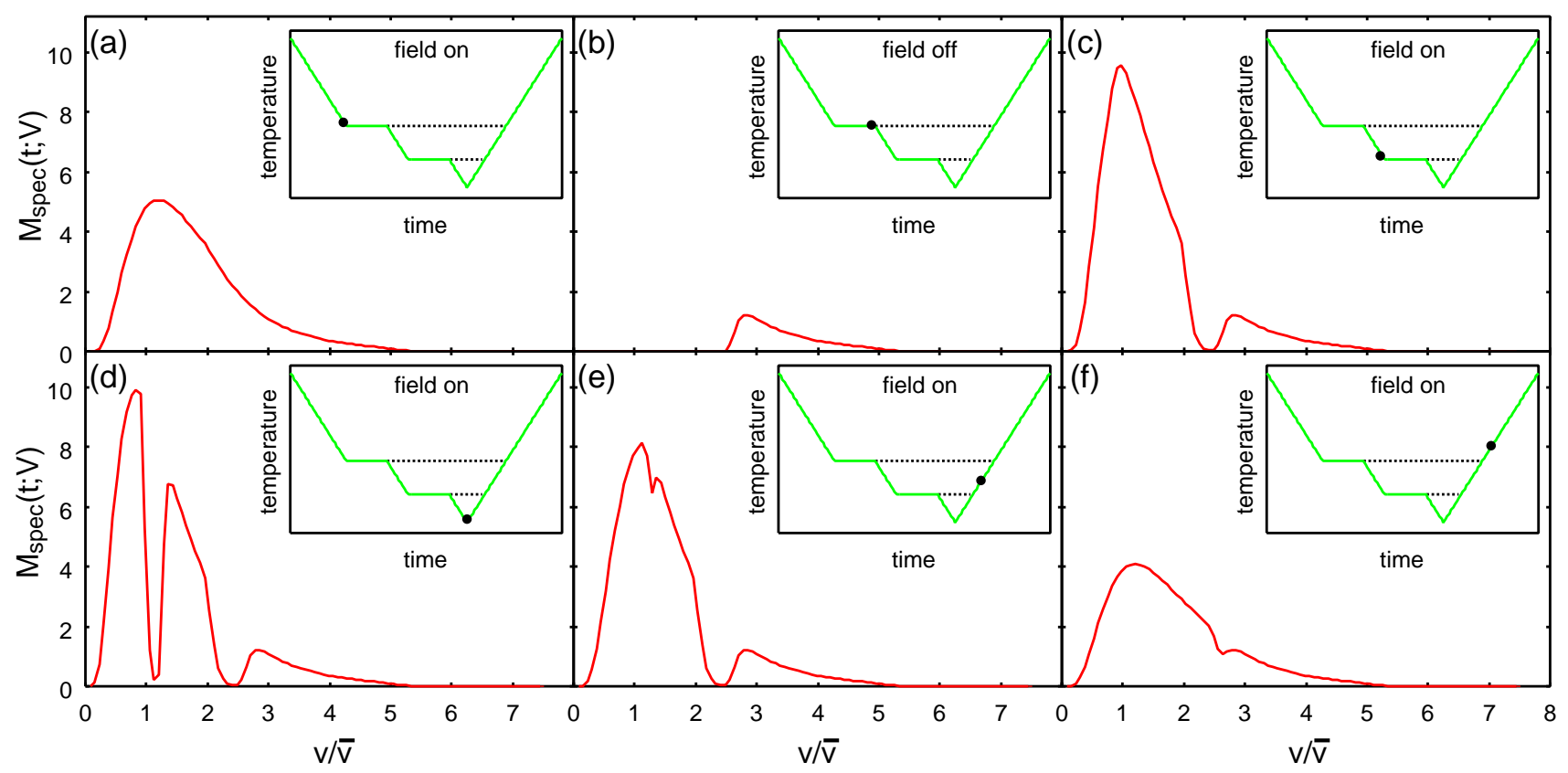

FIG. 5: (Color online) M-spectra at six representative states which are indicated in Fig. 4 by arrows. The point in each inset also shows the time of the measurement.

$T_{\mathrm{m}}$. Since the blocking volume $V_{\mathrm{B}}$ at $T_{2}$ is smaller than that at $T_{\mathrm{m}}$, the superspins which were dynamically active at $T_{\mathrm{m}}$ are frozen in the second stage at $T_{2}$, while the dynamically active superspins at $T_{2}$ do not change because they were already equilibrated (depolarized) by the firststage aging at $T_{\mathrm{m}}$. Hence $M_{\mathrm{TRM}}$ does not change at all in the second stage (squares in Fig. 3). The shape of the $M$-spectrum in this stage is essentially the same as that shown in Fig. (5), below. After the system comes back to $T_{\mathrm{m}}$ the relaxation of $M_{\mathrm{TRM}}$ resumes from the value at the end of the first-stage. If the field is applied in the second stage of the above protocol, the superparamagnetic and dynamically active superspins at $T_{2}$ respond to it. The $M$-spectrum at the end of this stage is essentially the same as that in Fig. 5(c). The induced magnetization in the second stage almost immediately disappears in the last stage at $T_{\mathrm{m}}$ since the superspins which carried the excess magnetization are rapidly equilibrated (depolarized) at the higher temperature. In the positivetemperature cycling with $T_{2}=0.042$ under $h=0$, superspins which are blocked at $T_{\mathrm{m}}$ but not at $T_{2}$ (i.e. superspins of nanoparticles whose volume is larger than $V_{\mathrm{B}}$ at $T_{\mathrm{m}}$ but smaller than $V_{\mathrm{B}}$ at $T_{2}$ ) are rapidly depolarized in the second stage. They are frozen as depolarized after changing the temperature back to $T_{\mathrm{m}}$, and thus $M_{\mathrm{TRM}}$ remains constant at a much smaller value. The significant relaxation is expected to resume at a time scale when the isothermal $M_{\mathrm{TRM}}$ at $T_{\mathrm{m}}$ reaches this small value. These features have been in fact observed by Sun et al $\frac{38}{3}$ in a permalloy nanoparticle system.

Lastly let us discuss the peculiar memory effect in Fig. 2 of Sun et al ${ }^{38}$. They introduce intermittent stops, at $T_{i}$, in the FC process and at the same time they cut off the field, let the system relax by a certain period $t_{i}$, and then resume the FC process. When the system is reheated after reaching a certain low temperature, the magnetization curve clearly manifests that the system keeps memories imprinted by the preceding FC process. We have applied the same protocol to our simple model of superparamagnets, and have reproduced qualitatively identical results to theirs as shown in Fig. 4

It is clarified in Fig. 5 that this peculiar memory effect originates from the blocking of superspins by demonstrating the $M$-spectra of some representative instants of the process. After the first stop at $T=T_{1}$ under $h=0$, the $M$-spectrum of Fig. (5) (b) tells us that the blocking volume $V_{\mathrm{B} 1}$ is around 3.0, namely, the superspins of nanoparticles with $V \lesssim V_{\mathrm{B} 1}$ are completely equilibrated (depolarized), while the frozen superspins of nanoparticles with $V \gtrsim V_{\mathrm{B} 1}$ are still blocked at $T=T_{1}$ after the waiting time. As the FC process is resumed, the memory of the first stop at $T=T_{1}$ is imprinted as a dip at $V \simeq V_{\mathrm{B} 1}$ in the $M$-spectrum [Fig. [5(c)], since that part of the $M$ spectrum is well blocked during the aging at significantly lower temperatures than $T_{1}$. Similarly, by the second stop at $T=T_{2}$ and recooling afterwards, another dip at $V_{\mathrm{B} 2} \simeq 1.3$ is imprinted in the $M$-spectrum as seen in Fig. 国(d). In the reheating process, Fig. 囵(e) and (f) illustrate that the frozen part of the $M$-spectrum melts starting from small $V$. The consequence is nothing but the memory effect reported by Sun et al. 


\section{Experiments on a superparamagnet}

In order to clarify how far our simple model captures the essence of real superparamagnets, we perform experiments on a model superparamagnet, namely natural horse-spleen ferritin 42.43 It is an iron-storage protein, and has a spherical cage $8 \mathrm{~nm}$ in diameter containing polydispersive cores of antiferromagnetic ferrihydrite 54,55 Each core has a small magnetic moment of $\sim 300 \mu_{\mathrm{B}}$ due to its uncompensated spins $\stackrel{42.56}{\text { Figure } 6}$ shows the result of the memory experiment with the same protocol as that in Fig. 4 It is clear from the figure that this superparamagnet also exhibits the same memory effect as that observed by Sun et al. In fact, this memory behavior is also observed in other superparamagnets 40.41 . The FCM without stops is shown in the inset of Fig. [6] We see that the FCM increases monotonically with decreasing temperature. As we discussed in the last paragraph of subsection $\llbracket B$ this is a typical feature of superparamagnets.

Figure 7 shows relaxation of the TRM susceptibility with the same protocol as that in Fig. 2(B). We clearly see that the TRM exhibits a similar $t_{\mathrm{w}}$ dependence to that in our simple model of superparamagnets. We have also checked a tendency that the peak of the relaxation rate $S(t) \equiv-h^{-1} d \log M / d \log t$ shifts to larger times with increasing $t_{\mathrm{w}}$, although the data are a bit too noisy to clarify whether the peak is located around $t_{\mathrm{w}}$ or not.

We have also done memory experiment in the genuine ZFC protocol. ${ }^{53}$ In this experiment, we measure $\chi_{\mathrm{ZFC}}$ which includes intermittent stops in the ZFC process and $\chi_{\mathrm{ZFC}}^{\mathrm{ref}}$ without such stops. The stopping temperatures are $9 \mathrm{~K}$ and $7 \mathrm{~K}$, and the period of intermittence is $10^{4} \mathrm{~s}$ at each temperature. Note that the stopping temperatures

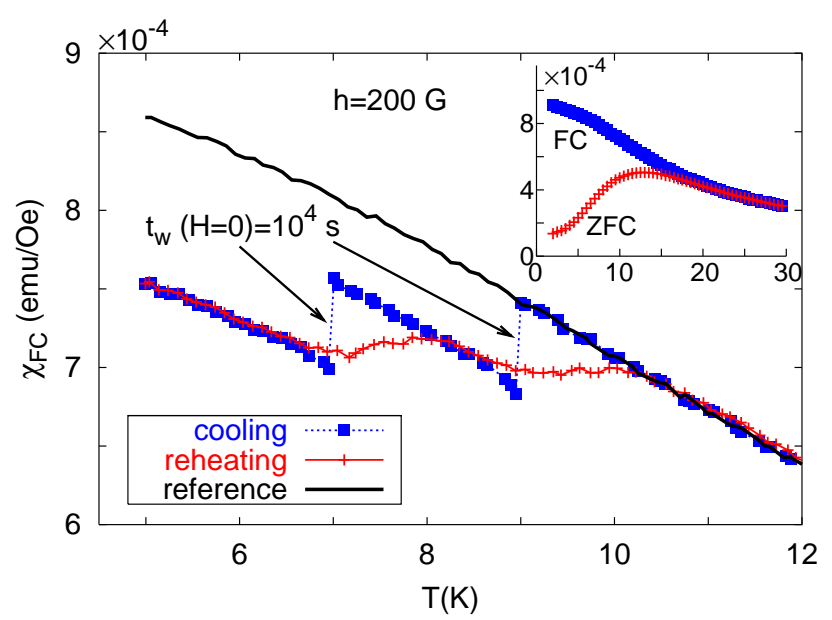

FIG. 6: (Color online) FC susceptibility of the ferritin with the same protocol as that in Fig. 4 The field is cut during the intermittent stops of the cooling at $T=9 \mathrm{~K}$ and at $T=7 \mathrm{~K}$ for $10^{4} \mathrm{~s}$ at each temperature. The cooling (and reheating) rate is $1.7 \times 10^{-3} \mathrm{~K} / \mathrm{s}$. The inset shows the $\mathrm{ZFC}$ and the FC susceptibilities vs temperature. are well below the blocking temperature $T_{\mathrm{B}} \approx 13 \mathrm{~K}$ (see the inset of Fig. 6). The cooling (and reheating) rate is the same as that in Fig. 6. The result of the experiment is that there is no significant difference between $\chi_{\mathrm{ZFC}}$ and $\chi_{\mathrm{ZFC}}^{\mathrm{ref}}$ at any temperatures (not shown), i.e., no memory is imprinted by the aging under zero field. This is also the expected result for superparamagnets.

\section{SLOW DYNAMICS IN SUPERSPIN GLASSES}

Various memory experiments are performed on a dense Fe-N ferromagnetic nanoparticle system which has been shown to be a superspin glass ${ }^{28.36 .44 .45}$ Figure 8 shows the result of the memory experiment following the protocol as that in Fig. 4. At the intermittent stops of the FC process, while the field is set to zero, the value of the magnetization decreases. On the subsequent reheating, the magnetization value in the preceding cooling process is recovered, for each stop, at a temperature a bit above that of the stop. At a glance, the memory effect in this superspin glass is qualitatively the same as that in superparamagnets indicating a similar origin of the effect. Another interesting observation in Fig. 8 is that the FCM of the Fe-N system after resuming the cooling behaves almost in parallel to the FCM without the intermittent stops (reference curve) though its absolute magnitude is significantly smaller than the latter. This feature is also seen for the superparamagnets as shown in Figs. 4 and 6 and so it suggests that the mechanism behind the memory effect is also common.

Now let us go into further comparisons between the results so far obtained for the superparamagnets and those

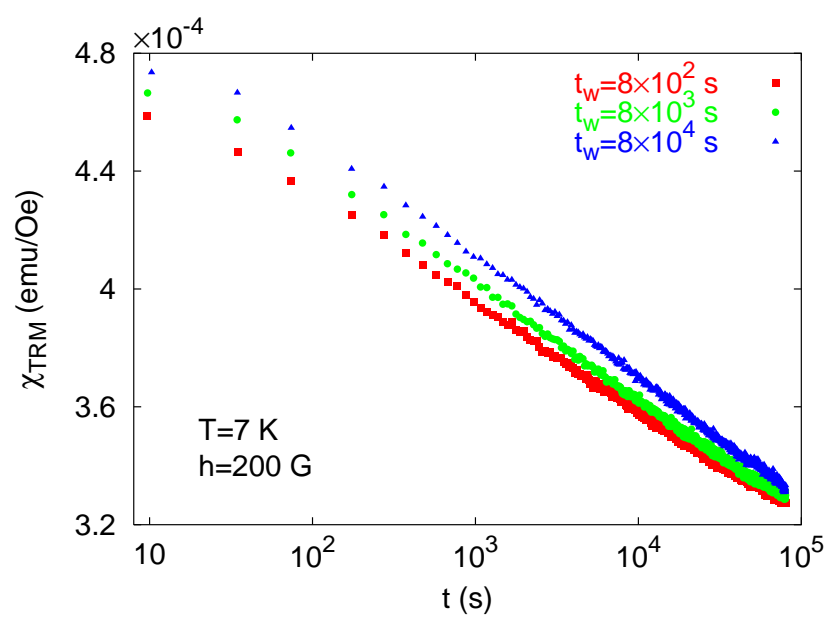

FIG. 7: (Color online) Relaxation of the TRM susceptibility of the ferritin. After the system is cooled to $T=7.0 \mathrm{~K}$ under a 200 Oe field at a rate of $0.17 \mathrm{~K} / \mathrm{s}$, it is kept at the temperature under the field for $t_{\mathrm{w}}$, and then the field is cut and the magnetization decay is measured as a function of the elapsed time $t$ after the field is cut. The waiting time $t_{\mathrm{w}}$ is $8 \times 10^{2}$, $8 \times 10^{3}$ and $8 \times 10^{4}$ (from left to right). 


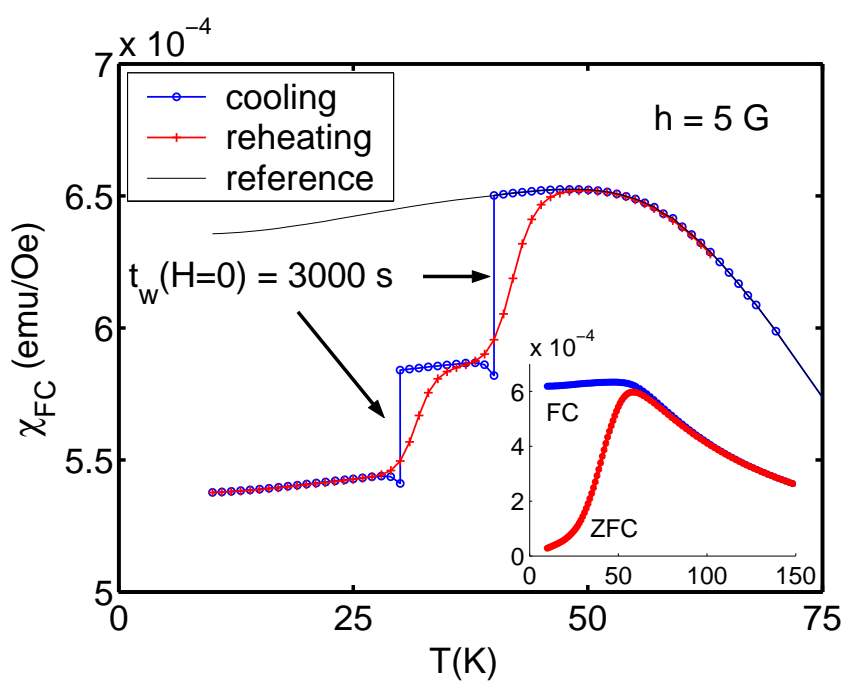

FIG. 8: (Color online) FC susceptibility of the Fe-N system with the same protocol as that in Fig. 4 The critical temperature of the sample is around $60 \mathrm{~K}{ }^{36}$ The field is cut during the intermittent stops of the cooling at $T=40 \mathrm{~K}$ and at $T=30 \mathrm{~K}$ for $3000 \mathrm{~s}$ at each temperature. The cooling (and reheating) rate is $0.01 \mathrm{~K} / \mathrm{s}$. The inset shows the $\mathrm{ZFC}$ and the FC susceptibilities vs temperature.

for the superspin glass. One significant difference between the two is seen in the behavior of the reference FCM without the intermittent stops. The FCM of the $\mathrm{Fe}-\mathrm{N}$ system does not increase but even decreases as the temperature is decreased. According to the argument in the last paragraph of subsection $\amalg B$ this implies that the Fe- $\mathrm{N}$ system is in fact not a superparamagnet also in this respect. Actually the nearly constant FCM is considered to be a typical property of ordinary spin glasses. A further important phenomenon which is peculiar to superspin glasses is memory effect in the genuine ZFC protocol. Figure 9 shows an experimental result of the Fe-N system where the difference between the ZFCM's with and without an intermittent stop at $T_{\mathrm{s}}$ in the cooling process is presented. The difference is clearly observed as a $\operatorname{dip}$ at $T \simeq T_{\mathrm{s}}$.

Now let us discuss possible theoretical interpretations of these experimental results. The first theoretical model we consider is the random energy model (REM) $49,50,51$ The REM consists of a huge number of states. The barrier energy $E_{\mathrm{B}}$, which the system needs to overcome in order to go to a new state, is assigned to each state randomly and independently according to the exponential distribution $\rho\left(E_{\mathrm{B}}\right)=1 / T_{\mathrm{c}} \exp \left[-E_{\mathrm{B}} / T_{\mathrm{c}}\right]$. Since the average relaxation time $\langle\tau\rangle=\int_{0}^{\infty} \mathrm{d} E_{\mathrm{B}} \rho\left(E_{\mathrm{B}}\right) \tau_{0} \exp \left(E_{\mathrm{B}} / T\right)$ diverges below $T_{\mathrm{c}}$, the REM shows various memory and aging behavior in the low temperature phase 50.51 .57 Let us now see to what extent the experimental results shown in this section are reproducible by the REM. First, Fig. 10] shows the result with the same protocol as that in Fig. 4. The result is qualitatively rather similar to that of the Fe-N system shown in Fig. 8 In particular, it

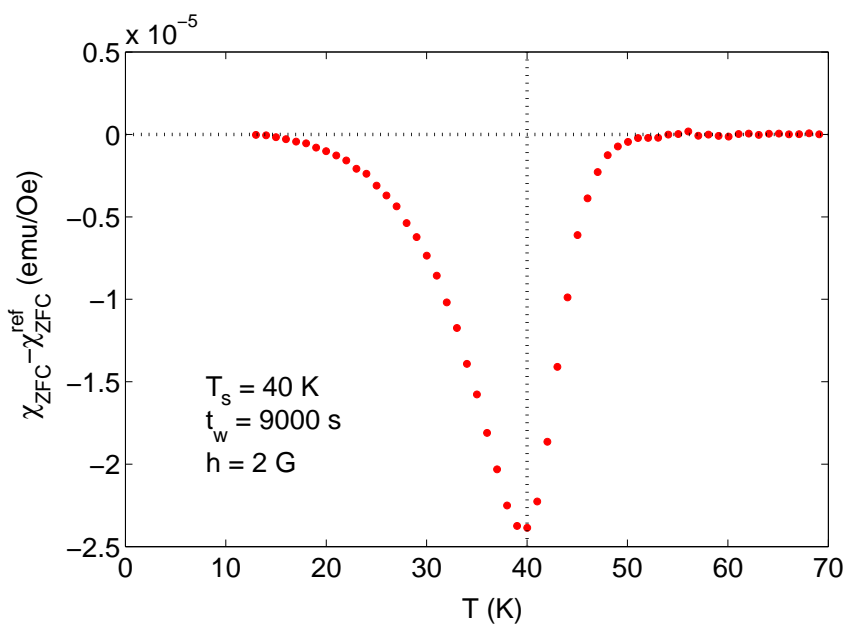

FIG. 9: (Color online) Difference of the ZFC susceptibility of the the Fe- $\mathrm{N}$ system. The ZFC process is intermitted at $T=40 \mathrm{~K}$ for $9000 \mathrm{~s}$ in the measurement of $\chi_{\mathrm{ZFC}}$, while $\chi_{\mathrm{ZFC}}^{\mathrm{ref}}$ is measured without such a stop. The cooling rate is $0.1 \mathrm{~K} / \mathrm{s}$, and the reheating rate is $0.01 \mathrm{~K} / \mathrm{s}$.

should be emphasized that the flatness of the FCM below the critical temperature, which can not be captured by our simple model of superparamagnets, is reproduced in the REM. Second, Fig. [11] shows the result of simulation which corresponds to the ZFC memory experiment in Fig. 9 Again, the result is qualitatively very similar to that in the experiment. A crucial property of the REM to understand this result is that the system goes into deeper and deeper states with higher and higher energy barriers as time progresses $\frac{50.58}{}$ Therefore, the typical energy barrier of the state in which the system is blocked depends on how long the system has been aged at a low temperature. Since it is more difficult for the system blocked in a state with a higher energy barrier to respond to the field, the difference of the typical energy barrier of the state in which the system is blocked with and without intermittent stop on cooling causes the dip in Fig. 11]

We have seen that the experimental results are well reproduced by the REM. However, the link between each state in the REM and an actual spin configuration in the system is not so clear. On the other hand, the droplet theory ${ }^{20.47}$ gives some insight into spin configurations in the (nonequilibrium) dynamics of real spin glasses. For example, after a spin glass is rapidly quenched in a field $h$ to a temperature $T$ below $T_{\mathrm{c}}$, spin-glass domains, or clusters, which are in local equilibrium with respect to $(T, h)$ are considered to grow. At a certain instance $t$ after the quench, clusters with various volumes $V_{\text {cluster }}$ or linear sizes $L\left(\sim V_{\text {cluster }}^{1 / d}\right)$ exist. We may think of their distribution $P\left(t ; V_{\text {cluster }}\right)$, analogously to $P(V)$ in the previous section. Furthermore, in the droplet theory, each cluster of a size $L$ is considered to flip by a thermal activation process whose mean energy barrier $B_{L}$ is a function of $L$ $\left(B_{L} \sim L^{\psi}\right.$ in the original droplet theory $\left.{ }^{20}\right)$. The thermally activated process governs the response of clusters 


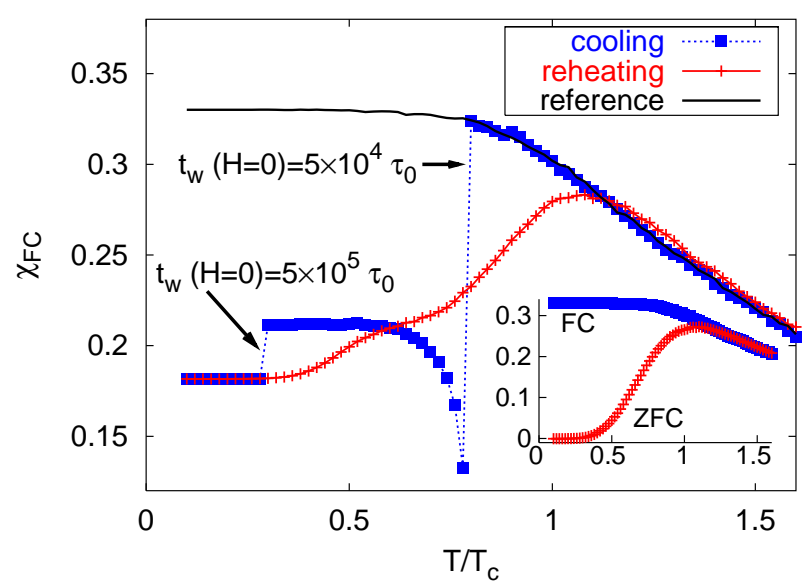

FIG. 10: (Color online) FC susceptibility of the REM with the same protocol as that in Fig. 4 The field is cut during the intermittent stops of the cooling at $T=0.8 T_{\mathrm{c}}$ for $5 \times 10^{4} \tau_{0}$ and at $T=0.3 T_{\mathrm{c}}$ for $5 \times 10^{5} \tau_{0}$, where $\tau_{0}$ is a microscopic time of the model. The cooling (and reheating) rate is $2 \times 10^{-5} T_{\mathrm{c}} / \tau_{0}$. The inset shows the $\mathrm{ZFC}$ and the FC susceptibilities vs temperature.

to an applied field. This situation is rather similar to the two-state description of the superparamagnet, and we may expect that the magnetization of the spin glass is also described by Eq.(6), though the functional form of $M(t ; V)$ has to be properly modified and $P(V)$ has to be replaced by a time-dependent distribution $P\left(t ; V_{\text {cluster }}\right)$. We also note that the above argument on an atomic spin glass can be directly applied to a superspin glass if an atomic spin in the former is replaced by a superspin in the latter.

An interesting prediction of the droplet theory is the instability of the equilibrium spin-glass phase under a static magnetic field $h$ of any strength. This is one of the fundamental issues which has been debated since the early stage of the spin-glass study and has not been settled yet. Quite recently, in numerical analysis of the fieldshift aging protocol, one of the present authors (HT) and Hukushima ${ }^{48}$ have found results which strongly support the prediction of the droplet theory. Here let us argue about our experimental results on the superspin glass from this point of view, namely, the FCM measured at $T<T_{\mathrm{c}}$ is not an equilibrium property under $h$ but due to the blocking of superspin clusters introduced above.

As noted before, the FCM of a superparamagnet increases with decreasing $T$. That of the present superspin glass, on the other hand, is nearly constant at $T \lesssim T_{\mathrm{c}}$ as seen in the inset of Fig. 8 The latter is naturally attributed to the expected fact that the free energy difference between the two states of a superspin cluster is given not only by the Zeeman energy but also by the residual interactions between the cluster and its surroundings (the stiffness energy of a cluster in the droplet theory). If the field strength is sufficiently small, which is the case of the present interest, the latter certainly dominates the Zee-

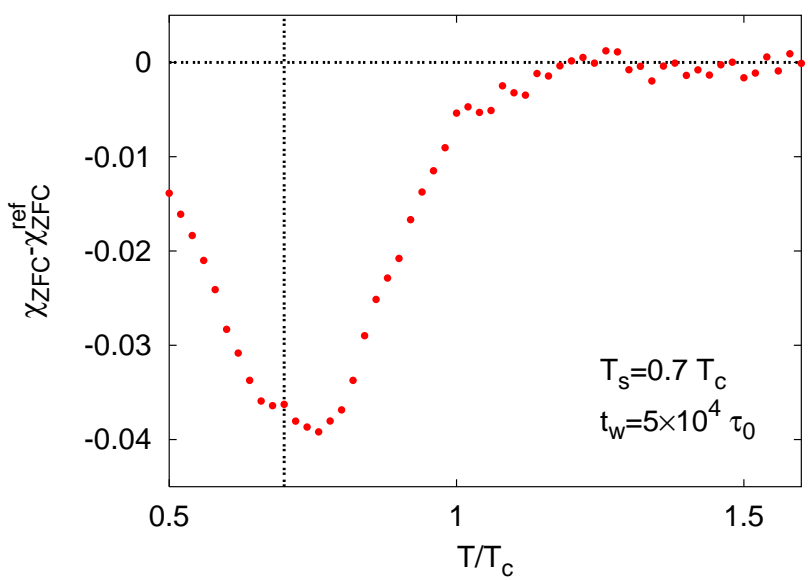

FIG. 11: (Color online) Difference of the ZFC susceptibility of the the REM. The ZFC process is intermitted at $T=0.7 T_{\mathrm{c}}$ for $5 \times 10^{4} \tau_{0}$ in the measurement of $\chi_{\mathrm{ZFC}}$, while $\chi_{\mathrm{ZFC}}^{\mathrm{ref}}$ is measured without such a stop. The cooling (and reheating) rate is the same as that in Fig. 10.

man energy. Therefore, when the cluster is blocked, its magnetization points either in parallel or antiparallel to the field direction. Consequently the branch of the FCM at $T \lesssim T_{\mathrm{c}}$ in Fig. 8 becomes nearly constant when the temperature is decreased.

By further inspection of Fig. 1 and the inset of Fig. 8 we notice that the FCM of a superparamagnet changes rather smoothly around the blocking temperature, while that of the superspin glass exhibits a kink-like shape at $T \sim T_{\mathrm{c}}$. The latter can be attributed to the time development of $P\left(t ; V_{\text {cluster }}\right)$ which is absent in a superparamagnet. In fact, in the droplet theory, the rates of growth of the spin-glass clusters and so of their barrier energy are expected to be most sensitive to a small change in temperature at $T \simeq T_{\mathrm{c}}$, since they are governed by the critical dynamics associated with the spin-glass transition at $T=T_{\mathrm{c}}$ under $h=0$. Consequently, even a small temperature decrease at this temperature range gives rise to an apparently sharper blocking of superspin clusters. At significantly lower temperatures than $T_{\mathrm{c}}$, the thermal activation process governs dynamics of superspins and yields an almost constant FCM as described just above.

Let us turn to memory effects in the genuine ZFC protocol, which are not observed in superparamagnets. As we mentioned above, at $T \lesssim T_{\mathrm{c}}$, sizes of clusters are growing as time elapses which gives rise to a history dependence of $P\left(t ; V_{\text {cluster }}\right)$ in the language of our two-states model. Since the change of $P\left(t ; V_{\text {cluster }}\right)$ proceeds even in a vanishing field, memory effects are observed even in the genuine ZFC protocol.

Lastly one comment is in order on possible differences in slow dynamics of superspin glasses and atomic spin glasses. As mentioned above, qualitative aspects of the two are considered to be almost common to each other. Quantitatively, however, the unit time of a superspin flip depend on $T$ and is much larger than the temperature 
independent atomic-spin flip time. This difference often causes apparent qualitative differences in the nonequilibrium phenomena in the two spin glasses because of the common experimental time window, $10^{1} \sim 10^{5} \mathrm{~s}$, which may differ very much when measured in the unit time of each system ${ }^{45}$

\section{SUMMARY}

We have studied dynamics of superparamagnets by investigating a simplified two-states model analytically and ferritin experimentally. As a consequence, we have found that

a) The TRM exhibits a $t_{\mathrm{w}}$-dependence, and the logarithmic time derivative of $\chi_{\mathrm{TRM}}\left(t, t_{\mathrm{w}}\right)$ has a peak around $t \approx t_{\mathrm{w}}$, as observed in spin glasses.

b) All the experimental results reported by Sun et al ${ }^{38}$ are qualitatively reproducible.

In superparamagnets, these aging and memory effects originate solely from a broad distribution of relaxation times which comes from that of the anisotropy energy barriers. The mechanism of these results is well understood by investigating the time dependence of the $M$ spectrum (the integrand in Eq.(6) ). Thus the aging and memory effects a-b) are not a sufficient proof for the existence of spin-glass dynamics.

We have also studied aging and memory effects in a dense Fe-N nanoparticle system (a superspin glass) experimentally. By comparing the results with those for superparamagnets, the following differences have been found:
1) The FCM of the Fe-N system does not increase but even decreases as the temperature is decreased, while the FCM of superparamagnets always increases with decreasing temperature.

2) In the Fe-N system, the genuine ZFCM also depends on the waiting time. Such a $t_{\mathrm{w}}$-dependence in the ZFCM is hardly expected in superparamagnets.

From the viewpoint of 1 ), we consider that the permalloy nanoparticle system studied by Sun et al. is closer to a superparamagnet, while the Fe-N system studied in the present work and the Co-Fe nanoparticle system studied by Sahoo et al ${ }^{30,31,37}$ are closer to a superspin glass. Lastly, we have argued that these two aspects peculiar to superspin glasses are qualitatively reproduced by the random energy model, and are well interpreted by the droplet theory in the field of the spin-glass study.

In conclusion, similarities as well as crucial differences in aging and memory effects in superparamagnets and superspin glasses have been clarified. In order to distinguish the two types of slow dynamics we have to choose appropriate aging protocols such as a ZFC process with intermittent stops of the cooling properly scheduled.

\section{Acknowledgments}

M.S. and P.E.J. acknowledge financial support from the Japan Society for the Promotion of Science. The present work is supported by a Grant-in-Aid for Scientific Research Program (\# 14540351) and NAREGI Nanoscience Project from the Ministry of Education, Culture, Sports, Science and Technology.
1 L. C. E. Struik, Physical aging in amorphous polymers and other materials. (Elsevier, Houston, 1978).

2 L. Lundgren, P. Svedlindh, P. Nordblad, and O. Beckman, Phys. Rev Lett. 51, 911 (1983).

3 L. Bellon, S. Ciliberto, and C. Laroche, (1999), condmat/9905160.

${ }^{4}$ L. Bellon, S. Ciliberto, and C. Laroche, Europhys. Lett. 51, 551 (2000).

${ }^{5}$ C. Rossel, Y. Maeno, and I. Morgenstern, Phys. Rev. Lett. 62, 681 (1989).

6 C. Josserand, A. V. Tkachenko, D. M. Mueth, and H. M. Jaeger, Phys. Rev. Lett. 85, 3632 (2000).

7 E. Vincent, J. P. Bouchaud, J. Hammann, and F. Lefloch, Phil. Mag. B 71, 489 (1995).

8 P. Nordblad and P. Svedlindh, in Spin Glasses and Random Fields, edited by A. P. Young (World Scientific, Singapore, 1998).

${ }^{9}$ K. Jonason et al., Phys. Rev. Lett. 81, 3243 (1998).

10 L. F. Cugliandolo and J. Kurchan, Phys. Rev. Lett. 71, 173 (1993).

11 J.-P. Bouchaud, L. Cugliandolo, J. Kurchan, and M. Mézard, in Spin Glasses and Random Fields, edited by
A. P. Young (World Scientific, Singapore, 1998).

12 H. Yoshino, A. Lemaître, and J.-P. Bouchaud, Eur. Phys. J. B. 20, 367 (2000).

13 M. Sasaki and K. Nemoto, J. Phys. Soc. Jpn 69, 2283 (2000).

14 T. Komori, H. Yoshino, and H. Takayama, J. Phys. Soc. Jpn. Suppl A 69, 228 (2000).

15 L. Berthier and J.-P. Bouchaud, Phys. Rev. B 66, 054404 (2002).

16 M. Mézard et al., J. Physique 45, 843 (1984).

17 M. Mézard et al., Phys. Rev. Lett. 52, 1156 (1984).

18 D. S. Fisher and D. A. Huse, Phys. Rev. Lett. 56, 1601 (1986).

19 A. J. Bray and M. A. Moore, Phys. Rev. Lett. 58, 57 (1987).

20 D. S. Fisher and D. A. Huse, Phys. Rev. B 38, 386 (1988).

21 A. Wills et al., Phys. Rev. B 62, R9264 (2000).

22 V. Dupuis et al., J. Appl. Phys. 91, 8384 (2002).

23 R. Mathieu, P. Svedlindh, and P. Nordblad, Europhys. Lett. 52 (4), 441 (2000).

${ }^{24}$ F. Alberich, P. Doussineau, and A. Levelut, J. Phys. I (France) 7, 329 (1997). 
25 F. Alberici-Kious et al., Phys. Rev. Lett. 81, 4987 (1998).

26 R. L. Leheny and S. R. Nagel, Phys. Rev. B 57, 5154 (1998).

27 T. Jonsson et al., Phys. Rev. Lett. 75, 4138 (1995).

${ }^{28}$ H. Mamiya, I. Nakatani, and T. Furubayashi, Phys. Rev. Lett. 82, 4332 (1999).

29 P. Jönsson, M. F. Hansen, and P. Nordblad, Phys. Rev. B 61, 1261 (2000).

30 S. Sahoo et al., J. Phys. C 14, 6729 (2002).

31 S. Sahoo et al., Phys. Rev. B 67, 214422 (2003).

32 P. E. Jönsson, Adv. Chem. Phys. 128, 191 (2004).

33 X. Batlle and A. Labarta, J. Phys. D: Appl. Phys. 35, R15 (2002).

34 W. Luo, S. R. Nagel, T. F. Rosenbaum, and R. E. Rosensweig, Phys. Rev. Lett. 67, 2721 (1991).

35 T. Jonsson, P. Svedlindh, and M. F. Hansen, Phys. Rev. Lett. 81, 3976 (1998).

${ }^{36}$ H. Mamiya and I. Nakatani, Nanostruct. Mater. 12, 859 (1999).

37 S. Sahoo et al., Phys. Rev. B 65, 134406 (2002).

38 Y. Sun, M. B. Salamon, K. Garnier, and R. S. Averback, Phys. Rev. Lett. 91, 167206 (2003).

39 M. Sasaki, P. E. Jönsson, H. Takayama, and P. Nordblad, (2003), cond-mat/0311264 (to be published).

40 R. K. Zheng and X. X. Zhang, (2004), cond-mat/0403368.

41 S. Chakravarty et al., (2004), cond-mat/0403574.

42 S. H. Kilcoyne and R. Cywinski, J. Magn. Magn. Mater. 140-144, 1466 (1995).

43 H. Mamiya, I. Nakatani, and T. Furubayashi, Phys. Rev. Lett. 88, 067202 (2002).

${ }^{4}$ H. Mamiya, I. Nakatani, and T. Furubayashi, Phys. Rev.
Lett. 80, 177 (1998).

45 P. E. Jönsson, H. Yoshino, H. Mamiya, and H. Takayama, (2004), cond-mat/0405276.

46 G. Parisi, Phys. Rev. Lett. 50, 1946 (1983), and references therein.

47 D. S. Fisher and D. A. Huse, Phys. Rev. B 38, 373 (1988).

${ }^{48}$ H. Takayama and K. Hukushima, (2003), condmat/0307641.

49 B. Derrida, Phys. Rev. B 24, 2613 (1981).

50 J. P. Bouchaud, J. Phys. I France 2, 1705 (1992).

51 J.-P. Bouchaud and D. Dean, J. Phys. I, France 5, 265 (1995).

52 I. Klik, C.-R. Chang, and J. Lee, J. Appl. Phys. 75, 5487 (1994).

53 R. Mathieu, P. Jönsson, D. N. H. Nam, and P. Nordblad, Phys. Rev. B 63, 092401 (2001).

54 D. D. Awschalom et al., Phys. Rev. Lett. 68, 3092 (1992).

55 P. M. Harrison, F. A. Fischbach, T. G. Hoy, and G. H. Haggis, Nature (London) 216, 1188 (1967).

56 S. A. Makhlouf, F. T. Parker, and A. E. Berkowitz, Phys. Rev. B 55, R14717 (1997).

57 M. Sasaki and K. Nemoto, J. Phys. Soc. Jpn 69, 3045 (2000).

58 M. Sasaki and K. Nemoto, J. Phys. Soc. Jpn 69, 2642 (2000).

59 More explicitly, the master equation (1) is solved by changing the temperature stepwise with a step of $\Delta T=4.2 \times$ $10^{-3}$ and at each temperature we let the system relax for a period of $\Delta t=1.0 \times 10^{10} \tau_{0}$. 